Scheduled appointments for blood alcohol level (BAL) testing may only uncover the most dependent of those who continue to drink. Our transplant unit, with patient consent, conducts community based BAL testing without prior notice as well as breath testing at the time of admission for LT. We evaluated the role of this process in identifying individuals who continue to drink alcohol while claiming abstinence. An update of a retrospective analysis of all patients with a diagnosis of ALD, who were listed for LT from 2006 to 2011, was performed. Patients with a positive BAL and those removed from the WL were identified.

Results 206 abstinent patients with ALD were listed for LT after evaluation for risk of recidivism. Four patients on the WL returned a positive BAL at planned appointments and eight upon testing without prior notice. Seven of the latter patients had previously returned negative tests at planned appointments and all were removed from the WL in line with UK national policy. One patient returned a positive test at the time of admission for LT. In addition, two patients admitted to drinking ( 1 with positive - but below the usual cut-off - BAL tests) and were removed from the WL.

Conclusion Random BAL testing is a useful tool in identifying ongoing hidden alcohol use in patients with ALD listed for LT. $6.8 \%$ of patients with ALD were removed from the WL due to ongoing alcohol ingestion. These patients may have damaged their grafts through ongoing alcohol ingestion and may recover without LT if they were to become abstinent. Identification of these patients is also important in maintaining public confidence in appropriate allocation of scarce donor livers.

Competing interests None declared.

\section{PTU-059 HEPATITIS C VIRUS INFECTION ABROGATES THE EFFECT OF LIVER TRANSPLANTATION ON NK CELL ACTIVATION}

doi:10.1136/gutjnl-2012-302514c.59

${ }^{1} \mathrm{~K}$ M Jamil, ${ }^{*} \mathrm{G}$ J Alexander, ${ }^{1} \mathrm{~K}$ C Cheent, ${ }^{3} \mathrm{~K}$ Agarwal, ${ }^{3} \mathrm{H}$ A Michael, ${ }^{4} \mathrm{~S}$ I Khakoo. ${ }^{1}$ Hepatology, Imperial College London, London, UK; ${ }^{2}$ Hepatology, Addenbrooke's Hospital, Cambridge, UK; ${ }^{3}$ Institute of Liver Studies, Kings College Hospital, London, UK; ${ }^{4}$ Hepatology, University of Southampton, Southampton, UK

Introduction Hepatitis $\mathrm{C}$ virus (HCV) infection is the leading cause for liver transplantation (LT) and recurrence post LT is universal. The role of NK cells in this context is poorly understood, in relation to both alloreactivity and antiviral response. This study investigates whether there are differences in NK cell activation between individuals transplanted for $\mathrm{HCV}$ and those transplanted for non-HCV indications.

Methods Whole blood was collected from 28 liver transplant patients (18 HCV, 10 non-HCV) and 16 controls. The transplant patients were all on calcineurin inhibitor-based immunosuppression. NK cells from the peripheral blood were analysed for expression of the cell surface inhibitory receptors CD158a/b (killer cell immunoglobulin-like receptors (KIR) specific for HLA-C), and the activating receptors NKp30, NKp46 and NKG2D. Following overnight incubation with IL-15 NK cell function was assessed using a flow cytometry-based killing assay.

Results Compared with controls, there was significantly reduced expression of NKp30 and NKp46 in non-HCV LT patients $(p<0.001$ for both, Student $t$ test), but no reduction in activating receptor expression in individuals transplanted for HCV. There was no segregation of activating receptor expression with expression of specific KIR. The functional assays correlated with the phenotyping data, with significantly reduced NK cell killing of target cells in nonHCV LT patients compared with controls and with HCV LT patients ( $p=0.02$ and 0.01 respectively, Student t test).
Conclusion The recipient NK cell response to liver transplantation is altered in individuals with HCV infection. In the non-HCV setting, we have demonstrated down-regulation of NK activation which may facilitate tolerance. However in the presence of HCV, NK cell function remains intact. We hypothesise that this phenomenon contributes to allograft inflammation and the poorer outcomes observed in LT for HCV.

Competing interests None declared.

\section{PTU-060 ENTERAL OR PARENTERAL FEEDING IN INTESTINAL GRAFT DYSFUNCTION: ANY CLUES FROM SERUM CITRULLINE?}

doi:10.1136/gutjnl-2012-302514c.60

${ }^{1} \mathrm{M}$ O'Connor, ${ }^{*}{ }^{2} \mathrm{~A}$ Vaidya, ${ }^{2} \mathrm{~L}$ Smith, ${ }^{2} \mathrm{P}$ Friend. ${ }^{1}$ Nutrition \& Dietetics, Oxford, UK; ${ }^{2}$ Oxford Transplant Centre, Oxford, UK

Introduction Citrulline has been successfully used as a marker for intestinal graft dysfunction after intestinal transplantation. ${ }^{1}$ We have extended its use to direct enteral vs parenteral feeding in the early post operative period as well as during graft dysfunction presenting as high volume stoma effluent.

Methods Weekly serum citrulline concentrations were used in directing nutrition in all recipients of intestinal grafts. A cut off of $13 \mu \mathrm{mol} / 1$ was used. Patients with citrulline levels $<13 \mathrm{mmol} / \mathrm{l}$ in the post operative period were kept on low volume enteral feed and maintained on TPN. Once the levels were above $13 \mathrm{mmol} / \mathrm{l}$, enteral feeds were advanced to meet target rate and TPN independence was achieved. A similar pattern was followed with patients presenting with high output stomal effluent. These citrulline levels were then matched with histopathological diagnosis.

Results From October 2008, nine patients underwent a small bowel transplant at the Oxford transplant centre. Mean citrulline levels week one after transplantation were $15 \mathrm{mmol} / \mathrm{l}$ (range 12-17). Endoscopic biopsies in the first week showed signs of ischaemia reperfusion with significant oedema in the submucosa of the transplanted ileum. All these patients were maintained on TPN and had enteral feed (Peptisorb) at $30 \mathrm{ml} / \mathrm{h}$. As mean citrulline levels increased patients progressed to full feed and TPN discontinued. Four patients presented with intestinal dysfunction after discharge from the hospital. These were all commenced on TPN if the citrulline level fell below $13 \mathrm{mmol} / \mathrm{l}$. The first, had a citrulline of $19 \mathrm{mmol} / \mathrm{l}$ on presentation and. Biopsy demonstrated acute rejection. The second, presented with a high stoma output with a citrulline level of $9 \mathrm{mmol} / \mathrm{l}$. Biopsy results revealed increase in mitotic figures as well as apoptosis and loss of tips of villi architecture. The third patient presented with a high stomal output and a citrulline of $18 \mathrm{mmol} / \mathrm{l}$. Citrulline fell further to $9 \mathrm{mmol} / \mathrm{l}$ The fourth patient presented with a high output and citrulline level of $13 \mathrm{mmol} / \mathrm{l}$, dropping further to $6 \mathrm{mmol} / \mathrm{l}$. His biopsy revealed dense mitotic activity in the face of increased apoptosis. He recovered in a span of 30 days with subsequent citrulline showing a rise. He was then weaned off TPN and commenced on diet. Mean time to graft dysfunction from transplantation was 240 days (range 46-450).

Conclusion Firstly serum citrulline is a good marker to direct nutritional therapy in the early post transplant period as well as during graft dysfunction.

Competing interests None declared.

\section{REFERENCE}

1. Crenn $\mathbf{P}$, et al. Postabsorptive plasma citrulline concentration is a marker of absorptive enterocyte mass and intestinal failure in humans. Gastroenterology 2000;119:1496. 